\title{
Biomarkers of disease activity in patients with chronic spontaneous urticaria
}

\author{
Aleksandra Plavsic ${ }^{1,2}$, Vesna Tomic-Spiric ${ }^{1,2}$, Snezana Arandjelovic ${ }^{1,2}$, Rada Miskovic ${ }^{1,2}$, Milan Dimitrijevic ${ }^{2}$, \\ Aleksandra Peric-Popadic ${ }^{1,2}$
}

${ }^{1}$ Department of Internal Medicine, Faculty of Medicine, University of Belgrade, Belgrade, Serbia ${ }^{2}$ Clinic of Allergy and Clinical Immunology, University Clinical Centre of Serbia, Belgrade, Serbia

Adv Dermatol Allergol 2021; XXXVIII (6): 1017-1022

DOI: https://doi.org/10.5114/ada.2021.112276

\begin{abstract}
Introduction: Previous studies have examined biomarkers of coagulation, inflammation and immunity in chronic spontaneous urticaria (CSU), but no recommended biomarkers for disease activity have been established yet. Aim: To find the relationship between certain laboratory parameters and disease activity in patients with CSU. Material and methods: Serum concentrations of D-dimer, C-reactive protein (CRP), C3, C4, and prothrombin time (PT), activated partial thromboplastin time (aPTT) values were measured in 44 CSU patients and compared with 33 healthy controls. Correlation between biomarkers and urticaria activity score during 7 consecutive days (UAS7) was calculated.

Results: Our study included 44 CSU patients (38 females and 6 males), mean age of 50.4 years and the average disease duration of 3.1 years. Based on UAS7, 23 (52.3\%) CSU patients had mild urticaria, 8 (18.2\%) well-controlled, 7 (15.9\%) moderate and 6 (13.6\%) severe urticaria. Fourteen (31.8\%) patients had elevated CRP, 21 (47.7\%) had elevated D-dimer and 14 (13.6\%) CSU patients had elevated C4 levels. Patients with CSU had statistically significant elevated D-dimer, CRP and PT as compared with controls ( $p=0.007, p=0.005$ and $p=0.029$, respectively). There was no correlation between PT, aPTT, D-dimer, CRP, C3 and disease activity. Statistically significant differences in C4 levels between patients with severe and well-controlled, mild, moderate urticaria were determined $(p=0.003)$. Conclusions: CRP, D-dimer, and PT may be considered as biomarkers for distinguishing patients with CSU from controls. The C4 levels correlate with disease activity and may be useful as a potential biomarker of disease activity.
\end{abstract}

Key words: chronic spontaneous urticaria, biomarkers, disease activity.

\section{Introduction}

Chronic spontaneous urticaria (CSU) is a heterogeneous disease characterized by short lived wheals with or without angioedema that last for more than 6 weeks. The diagnosis is based on clinical evaluation of typical skin changes. The wheals vary in intensity and duration, causing a significant influence on the quality of life and a substantial socio-economic burden. The aetiology and pathogenesis of CSU are still not completely understood. Autoimmunity, inflammation and coagulation processes are considered to play an important role in CSU pathogenesis [1].

Chronic spontaneous urticaria is associated with variable duration and response to therapy, so it is important to find biomarkers to assess disease activity that could help predict the disease duration and best treatment option. It is recommended to use urticaria activity score during 7 consecutive days (UAS7) as a disease assessment tool, a system that scores key urticaria signs and symptoms (wheals and pruritus), documented by the patient [2]. This is a highly subjective method, so disease activity is evaluated by the patient. There are no currently recommended biomarkers for disease activity, duration and treatment response in CSU patients.

Several studies have shown that patients with CSU have elevated levels of prothrombin fragment $1+2(\mathrm{~F} 1+2)$, D-dimer and FVIla and that these markers correlate with disease activity [3-7]. It is found that serum concentrations of C-reactive protein (CRP), IL-6 and matrix-metalloproteinase 9 (MMP-9) are increased in urticaria patients and that they correlate with disease activity [8-10]. Kolkhir et al. [11] have reviewed available literature on blood biomarkers for chronic urticaria and strong evidence was found for 10 biomarkers (D-dimer, CRP, MMP-9,

Address for correspondence: Aleksandra Plavsic Assist. Prof., Department of Internal Medicine, Faculty of Medicine, University of Belgrade, Koste Todorovica 2, 11000 Belgrade, Serbia, phone: +381 668300 735, e-mail: sandrony@yahoo.com Received: 16.06.2020, accepted: 13.07.2020. 
mean platelet volume (MPV), factor VIIa, F1+2, tumour necrosis factor, dehydroepiandrosterone sulphate and vitamin D) for distinguishing patients with CSU from healthy controls. Also, a significant association between CSU activity and D-dimer, F1+2, CRP, IL-6 and MPV was shown $[11,12]$.

\section{Aim}

Based on the results of previous studies that have found increased levels of certain biomarkers in CSU and correlation between biomarkers and disease activity, we aimed to evaluate some inflammatory (CRP), coagulation (prothrombin time (PT), activated partial thromboplastin time (aPTT), D-dimer) and immunological $(C 3, C 4)$ parameters in CSU patients, compare them to healthy controls and to investigate the relationship between biomarkers and disease activity.

\section{Material and methods \\ Patients}

We enrolled 44 consecutive patients treated from June 2017 to July 2018 at the Clinic for Allergy and Immunology, University Clinical Centre of Serbia, who fulfilled EAACI/GA $\left({ }^{2}\right)$ LEN/EDF/WAO criteria for CSU diagnosis (spontaneous wheals with or without angioedema appearance daily or almost daily for $\geq 6$ weeks, with no obvious external specific trigger). Exclusion criteria was the use of anticoagulation and antiplatelet therapy. The therapy for CSU was not stopped. The demographic data and personal history were recorded. The control group consisted of 33 healthy subjects. The study was approved by the local Ethical Committee and written consent was obtained from CSU patients and the controls (668/2).

\section{Assessment of disease activity}

Disease activity was assessed using UAS7. It quantifies the number of wheals (none: 0 , mild: 1 ( $<20$ lesions

Table 1. Characteristics of CSU patients

\begin{tabular}{lcc}
\hline Characteristics & N (44) & Percentage (\%) \\
\hline CSU without angioedema & 20 & 45.5 \\
\hline CSU with angioedema & 24 & 54.5 \\
\hline Elevated CRP & 14 & 31.8 \\
\hline Elevated D-dimer & 21 & 47.7 \\
\hline Increased C4 & 6 & 13.6 \\
\hline Well-controlled CSU & 8 & 15.9 \\
\hline Mild CSU & 23 & 52.3 \\
\hline Moderate CSU & 7 & 15.9 \\
\hline Severe CSU & 6 & 13.6 \\
\hline
\end{tabular}

CSU - chronic spontaneous urticaria, CRP - C-reactive protein. in $24 \mathrm{~h}$ ), moderate: 2 (20-50 lesions in $24 \mathrm{~h})$, severe: 3 (> 50 lesions in $24 \mathrm{~h}$ )) and pruritus intensity (none: 0 , mild: 1 (present but not annoying or troublesome), moderate: 2 (troublesome but not interfering with normal activity or sleeping) and intense: 3 (severe pruritus, which is sufficiently troublesome to interfere with normal daily activity or sleep)) on a daily basis for 7 consecutive days. Total score ranges from 0 to 42, allowing to classify disease activity as: well-controlled (0-6), mild (7-15), moderate (16-27), or severe (28-42).

\section{Laboratory tests}

Serum concentrations of CRP (turbidimetric, ARCHITECT), D-dimer (turbidimetric, BCS XP Siemens), C3 and C4 (turbidimetric, ARCHITECT), and PT and aPTT values (Sysmex CS-5100) were measured according to the manufacturer's instructions. The reference ranges for CRP were 0-5 mg/l, for D-dimer 0-0.5mg/l FEU, for PT 10-13 s, for aPTT 22-32 s, for C3 0.83-1.93 g/l, for C4 0.12-0.36 g/l, respectively.

\section{Statistical analysis}

Statistical analyses were performed using SPSS for Windows, version 20. We used descriptive statistical methods (both numerical and graphical tools including mean, mode/median, percentage) and analytical methods ( $T$-test, ANOVA, Spearman's rank correlation coefficient). In all tests, $p$-values $<0.05$ were considered significant.

\section{Results}

Forty-four patients, 38 (86.4\%) females and 6 (13.6\%) males, mean age of 50.4 (18-74) years and mean CSU duration of 3.1 years were enrolled in the study. Angioedema with urticaria was present in 24 (54.5\%) patients. Fourteen (31.8\%) patients had elevated CRP and $21(47.7 \%)$ had elevated D-dimer. Increased C4 levels were found in 6 patients. Prolonged PT was present in $1(2.3 \%)$ patient and prolonged aPTT also in one patient. In 1 patient, PT was decreased, and in $6.3 \%$ aPTT was decreased. Based on UAS7, more than half of CSU patients 23 (52.3\%) had mild urticaria, 8 (18.2\%) had wellcontrolled, 7 (15.9\%) moderate and 6 (13.6\%) of patients had severe urticaria (Table 1).

Patients with CSU had statistically significant elevated PT as compared with controls $(p=0.029)$. D-dimer was statistically significantly elevated in CSU patients as compared with controls ( $p=0.007$ ) (Figure 1 ). Also, CSU patients had statistically significant elevated CRP as compared with controls ( $p=0.005$ ) (Figure 2). There was no significant difference in aPTT between CSU patients and controls $(p=0.80)$.

There was no correlation between D-dimer and disease activity ( $\rho=0.293 ; p=0.054)$. Mean D-dimer in 


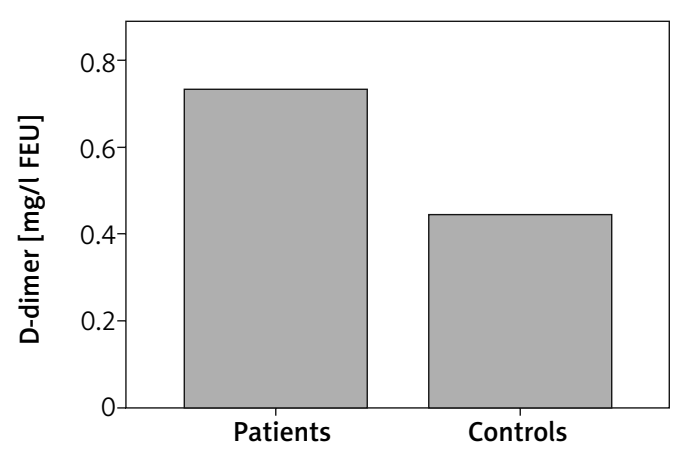

Figure 1. D-dimer concentrations in CSU patients and controls. CSU patients have statistically significantly elevated D-dimer as compared with controls $(p=0.007)$

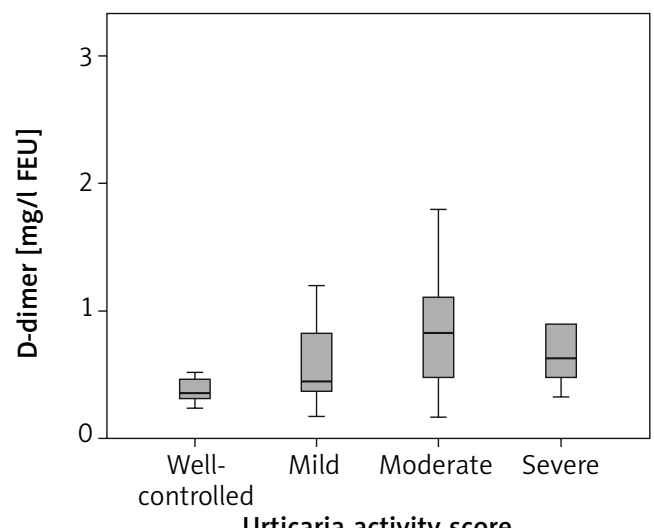

Figure 3. D-dimer levels in CSU patients with well-controlled, mild, moderate and severe urticaria. There was no difference in D-dimer between these groups $(p=0.49)$

patients with well-controlled CSU was $0.46 \mathrm{mg} / \mathrm{FEU}$, in mild urticaria $0.73 \mathrm{mg} / \mathrm{I} \mathrm{FEU}$, in moderate $0.85 \mathrm{mg} / \mathrm{I} \mathrm{FEU}$, and severe $0.98 \mathrm{mg} / \mathrm{FEU}$, but there was no statistically significant difference in D-dimer between these groups $(p=0.49)$ (Figure 3). No correlation was found between CRP, C3 and disease activity $(\rho=0.24 ; p=0.105$ and $\rho=0.037 ; p=0.813$, respectively). There were no statistical differences in CRP and in C3 levels between patients with well-controlled, mild, moderate and severe urticaria ( $p=0.244$ and $p=0.862$, respectively). Prothrombin time and aPTT did not correlate with disease activity $(\rho=-0.168 ; p=0.247$ and $\rho=-0,178 ; p=0.247$, respectively). No difference was found in PT and aPTT between patients with well-controlled, mild, moderate and severe urticaria ( $p=0.339, p=0.339$, respectively).

We found a correlation between $C 4$ levels and disease activity $(\rho=0.32 ; p=0.034)$. Analysis of variance has shown a statistically significant difference in C4 between patients with severe CSU and well -controlled, mild, moderate urticaria $(p=0.003)$. Further

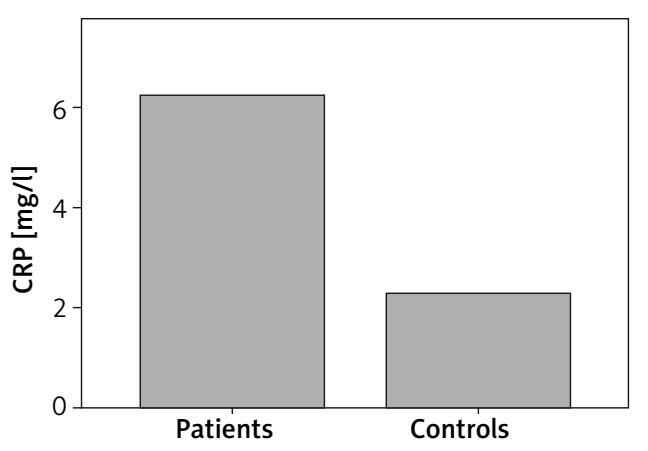

Figure 2. CRP concentrations in CSU patients and controls. CSU patients have statistically significantly elevated CRP as compared with controls $(p=0.005)$

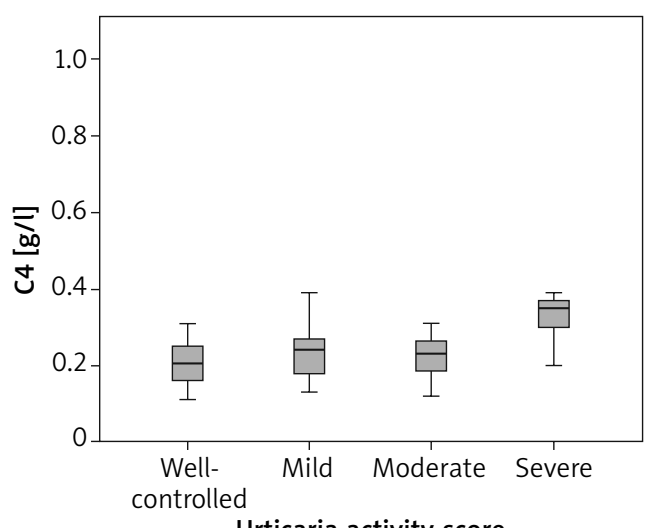

Figure 4. C4 levels in CSU patients grouped by disease activity. Patients with severe CSU have significantly elevated C4 as compared to moderate, mild and well-controlled CSU

analysis has shown that patients with severe CSU had significantly higher C4 levels as compared with patients with well-controlled, mild and moderate urticaria (Figure 4).

\section{Discussion}

Chronic spontaneous urticaria is characterized by specific skin changes that usually do not present a diagnostic problem. However, the duration of CSU is very unpredictable, recommended therapy is often not effective, the aetiology remains unknown despite all investigations done and the pathophysiological mechanism is still not fully understood. Considering all these, there is a need for identification of biomarkers that would help to assess disease activity and to predict duration of the disease and response to therapy [13].

In recent years, many research studies evaluated activation of coagulation in CSU patients. Elevated D-dimer was present in $47.7 \%$ of our patients and was statistically significantly higher as compared with controls. Other studies have also shown increased D-dimer levels in CSU 
patients compared with controls in CSU patients compared with controls [14-16]. D-dimer is a marker of fibrin production and degradation of stable fibrin and reflects coagulation activation and fibrinolysis. Increased levels in our study are suggestive of the role of the coagulation process in the development of urticaria. Takahagi et al. [17] have observed that D-dimer may be the most sensitive biomarker to detect coagulation abnormalities in chronic urticaria, but fibrin degradation product (FDP) may be the most useful biomarker for disease activity. Based on the remark that levels of FDP and D-dimer are not different in patients with chronic urticaria, acute urticaria and angioedema, authors have suggested that coagulation activation may be a common phenomenon, not specific for chronic urticaria and might be a result of mast cell activation, rather than a primary event in wheal formation.

Our results revealed a significant difference in mean PT between patients and controls. Prothrombin time presents the time needed for blood to coagulate when tissue factor (TF) is added and it evaluates the extrinsic (and common) pathways of coagulation (FI, FII, FV, FVII, FX). Our findings indicate the role of the extrinsic coagulation pathway in CSU that is initiated by TF expression. Previous studies have found that TF from eosinophils in chronic urticaria activates extrinsic coagulation processes, with conversion of prothrombin into thrombin [18]. Thrombin acts on fibrinogen and produces fibrin that is degraded into FDP and D-dimer. Also, thrombin increases vascular permeability causing dermal oedema and stimulates mast cell degranulation [19] and may activate protease activated receptor 1 on mast cells [20]. However, we did not find the difference in aPTT between patients and controls $(p=0.80)$. Kim et al. [21] have shown prolonged aPTT in patients with chronic urticaria, but PT did not differ between patients and controls. Tests for PT and APTT are important to evaluate initial pathways in coagulation, such as initiation of thrombin production and may indicate the importance of both intrinsic and extrinsic pathways in CSU.

We have found elevated CRP in $31.8 \%$ of CSU patients. Other studies have also shown increased CRP levels in 23-31\% of CSU patients [17, 22]. Our patients had statistically significant elevated CRP as compared with controls, that is shown in other studies as well [14, 15]. C-reactive protein is a sensitive marker of inflammation, and as such is used as a biomarker of disease activity in many diseases, so it is not specific for CSU. However, current recommendations for CSU suggest that CRP should be done in all chronic urticaria patients [2]. Kolkhir et al. [22] have retrospectively analysed data from 1253 chronic urticaria patients and they have found that CRP was significantly higher in nonresponders to antihistamines as compared to responders, so CRP could act as a potential biomarker for therapy response. Also, it has been suggested that measurements of CRP may be useful in clinical practice to evaluate therapy response and disease exacerbation as it correlates with disease activity and severity [14]. However, clinical use of CRP should be considered as some patients with CSU have other diseases in which CRP could be also increased.

We did not show the correlation between coagulation (PT, aPTT, D-dimer) and inflammation (CRP) markers and disease activity and no difference was found in these parameters between CSU patients with well-controlled, mild, moderate and severe urticaria. There was an increase in mean D-dimer levels in patients with different levels of disease activity: D-dimer concentration in wellcontrolled urticaria was $0.46 \mathrm{mg} / \mathrm{FEU}$, in mild urticaria $0.73 \mathrm{mg} / \mathrm{I} \mathrm{FEU}$, in moderate $0.85 \mathrm{mg} / \mathrm{l} \mathrm{FEU}$, and in severe it was $0.98 \mathrm{mg} / \mathrm{l} \mathrm{FEU}$. Even though the trend toward higher D-dimer in patients with more active disease was observed, and results were at the cut-off point of statistical significance $(p=0.054)$, no correlation between D-dimer and urticaria activity was shown. The results of most other studies have shown that D-dimer correlates with urticaria disease activity and severity $[4-7,15,17]$ as well as CRP $[8,9,14,16,17]$. Our results can be explained by the fact the most of our patients, 32 out of 44 , were taking therapy for CSU, which could affect clinical presentation, CRP and D-dimer concentrations and UAS7 scoring. Also, more than half of CSU patients (52.3\%) had mild urticaria, $18.2 \%$ had well-controlled, $15.9 \%$ had moderate and only $13.6 \%$ of patients had severe urticaria. In a study done in Poland, D-dimer, CRP, IL-6 concentrations were statistically higher in CSU patients as compared with controls, and a correlation between D-dimer and CRP, IL-6 and UAS was demonstrated [14]. All 58 patients in this study were without therapy for CSU and were classified into two groups based on modified UAS7 that was performed for 4 days: 8 had mild CSU (0-8 points) and 27 moderate-severe CSU (9-24 points). Many studies use UAS7 as a tool for disease activity evaluation, but there are no guidelines how to assess disease activity based on points gathered via this scoring system. It is not clear if disease activity reflects disease severity and vice versa as studies that evaluate biomarkers use both terms. This makes comparison of the results in different studies more difficult. In our study, we have classified CSU patients based on UAS7 into 4 groups: well-controlled, mild, moderate and severe CSU. Forty-four patients were enrolled and they were divided into 4 groups, so we can say that limitation of our study is the number of enrolled CSU patients.

We evaluated the role of the complement system in urticaria and have found a correlation between C4 levels and disease activity. Further analysis has shown a statistically significant difference in C4 between patients with severe and well-controlled, mild, moderate urticaria. Only patients with severe CSU had elevated mean C4 levels $(0.40 \mathrm{~g} / \mathrm{l})$ and patients with well-controlled, mild and moderate had normal C4 levels $(0.20 \mathrm{~g} / \mathrm{l}, 0.23 \mathrm{~g} / \mathrm{l}$ and $0.22 \mathrm{~g} / \mathrm{l}$, respectively). No correlation was found in C3 
levels and disease activity. Kasperska-Zajac et al. [23] have evaluated C3, C4 and CRP in 70 urticaria patients, all therapy free, and they have classified disease activity into three groups (mild, moderate and severe). They found that serum C3 and C4 concentrations were significantly increased in patients as compared with the healthy subjects and those with moderate-severe symptoms showed higher C3 and C4 concentrations as compared with mild CU patients and healthy subjects. However, $90 \%$ of patients with severe urticaria had normal C4 levels and 95\% had normal C3 levels, so despite severe disease activity, C3 and C4 concentrations were in normal range in most patients. They questioned the role of the complement system in urticaria, if increased serum C 3 and C4 concentrations present the biomarker of acute phase response activation or they play an active role in urticarial processes. Another study has shown that 18 out of 35 patients with urticaria had low C4 levels and an association between reduction in $\mathrm{C} 4$ and positive autologous serum skin test were found, but with no clear relationship between them [24]. The role of C3 and C4 has not been explored extensively in CSU patients. Previous studies have shown that $\mathrm{C} 5 \mathrm{a}$, part of the complement system, is involved in mast cells activation in the course of CU [25]. The complement system is composed of many proinflammatory proteins, $\mathrm{C} 4$ being the major protein of the classical cascade. Elevated C4 may be the result of increased synthesis in the liver in response to cytokines IL-1 , IL-6 or tumour necrosis factor, which are increased in active urticaria [26]. Regarding these findings and our results, C4 may be an important player in active CSU, however its exact role still remains unknown.

In recent years, great research effort has been done to find reliable biomarkers in CSU. The problems with biomarkers in CSU are their specificity, usefulness in clinical practice and availability. It is not clear if inflammation, autoimmunity, coagulation processes in CSU may overlap or one play a dominant part in pathogenesis. It has been shown that markers of inflammation (IL-6, CRP) and coagulation/fibrinolysis (D-dimer) are related in CSU, and that there may be an interaction between these biological systems [14]. This suggests the existence of different phenotypes in CSU. The role of the biomarker is to identify these different groups of patients, to classify them according to the underlying pathophysiological mechanism and to find the best therapy option. More studies are needed in the future to resolve these issues.

\section{Conclusions}

CRP, D-dimer, and PT may be considered as biomarkers for distinguishing patients with CSU from controls. However, CRP, D-dimer, aPTT and C3 do not correlate with disease activity, but their involvement in CSU should be considered and further explored. Our results indicate the role of C4 as a marker of disease activity, but more studies are needed to evaluate the role of the complement system in urticaria. C-reactive protein and C4 are inflammation markers and they are increased in CSU patients, so inflammation may have an important part in CSU pathogenesis.

\section{Acknowledgments}

The study was conducted at the Clinic of Allergy and Clinical Immunology, University Clinical Centre of Serbia, Belgrade.

\section{Conflict of interest}

The authors declare no conflict of interest.

\section{References}

1. Asero R, Tedeschi A, Marzano AV, et al. Chronic spontaneous urticaria: immune system, blood coagulation, and more. Expert Rev Clin Immunol 2016; 12: 229-31.

2. Zuberbier T, Aberer W, Asero R, et al. The EAACI/GA(2)LEN/ EDF/WAO Guideline for the definition, classification, diagnosis, and management of urticaria. Allergy 2018; 73: 1393-414.

3. Asero R, Tedeschi A, Riboldi P, et al. Plasma of patients with chronic urticaria shows signs of thrombin generation, and its intradermal injection causes wheal-and flare reactions much more frequently than autologous serum. J Allergy Clin Immunol 2006; 117: 1113-7.

4. Asero R, Tedeschi A, Riboldi P, et al. Severe chronic urticaria is associated with elevated plasma levels of D-dimer. Allergy 2008; 63: 176-80.

5. Rabelo-Filardi R, Daltro-Oliveira R, Campos RA. Parameters associated with chronic spontaneous urticaria duration and severity: a systematic review. Int Arch Allergy Immunol 2013; 161: 197-204.

6. Triwongwaranat D, Kulthanan K, Chularojanamontri L, et al. Correlation between plasma D-dimer levels and the severity of patients with chronic urticaria. Asia Pac Allergy 2013; 3: 100-95.

7. Wang F, Tang H, Xu JH, et al. Activation of the blood coagulation cascade is involved in patients with chronic urticaria. J Allergy Clin Immunol 2009; 123: 972-3.

8. Kasperska-Zajac A, Sztylc J, Machura E, et al. Plasma IL-6 concentration correlates with clinical disease activity and serum C-reactive protein concentration in chronic urticaria patients. Clin Exp Allergy 2011; 41: 1386-91.

9. Tedeschi A, Asero R, Lorini M, et al. Plasma levels of matrix metalloproteinase-9 in chronic urticaria patients correlate with disease severity and C-reactive protein but not with circulating histamine-releasing factors. Clin Exp Allergy 2010; 40: 875-81.

10. Kessel A, Bishara R, Amital A, et al. Increased plasma levels of matrix metalloproteinase-9 are associated with the severity of chronic urticaria. Clin Exp Allergy 2005; 35: 221-5.

11. Kolkhir P, André F, Church MK, et al. Potential blood biomarkers in chronic spontaneous urticaria. Clin Exp Allergy 2017; 47: 19-36.

12. Deza G, Ricketti PA, Gimenez-Aranau AM, et al. Emerging biomarkers and therapeutic pipelines for chronic spontaneous urticaria. J Allergy Clin Immunol Pract 2018; 6: 1108-17. 
13. Folci M, Heffler E, Canonica W, et al. Cutting edge: biomarkers for chronic spontaneous urticaria. J Immunol Res 2018; 2018: 5615109.

14. Grzanka R, Damasiewicz-Bodzek A, Kasperska-Zajac A. Interplay between acute phase response and coagulation/ fibrinolysis in chronic spontaneous urticaria. Allergy Asthma Clin Immunol 2018; 14: 27.

15. Baek YS, Jeon J, Kim JH, et al. Severity of acute and chronic urticaria correlates with D-dimer level, but not C-reactive protein or total IgE. Clin Exp Dermatol 2014; 39: 795-800.

16. Cugno M, Tedeschi A, Borghi A, et al. Activation of blood coagulation in two prototypic autoimmune skin diseases: a possible link with thrombotic risk. PLoS One 2015; 10: e0129456.

17. Takahagi S, Mihara S, Iwamoto K, et al. Coagulation/fibrinolysis and inflammation markers are associated with disease activity in patients with chronic urticaria. Allergy 2010; 65: 649-56.

18. Cugno M, Marzano AV, Asero R, et al. Activation of blood coagulation in chronic urticaria: pathophysiological and clinical implications. Intern Emerg Med 2010; 5: 97-101.

19. Asero R, Tedeschi A, Coppola R, et al. Activation of the tissue factor pathway of blood coagulation in patients with chronic urticaria. J Allergy Clin Immunol 2007; 119: 705-10.

20. Dugina TN, Kiseleva EV, Chistov IV, et al. Receptors ofthe PAR family as a link between blood coagulation and inflammation. Biochemistry 2002; 67: 65-74.

21. Kim JA, Kim S, Kim JE, et al. Activation of the intrinsic coagulation pathway in patients with chronic urticaria. Allergy Asthma Immunol Res 2015; 7: 476-82.

22. Kolkhir P, Altrichter S, Hawro T, et al. C-reactive protein is linked to disease activity, impact, and response to treatment in patients with chronic spontaneous urticaria. Allergy 2018; 73: 940-8.

23. Kasperska-Zajac A, Grzanka A, Machura E, et al. Increased serum complement C3 and C4 concentrations and their relation to severity of chronic spontaneous urticaria and CRP concentration. J Inflamm 2013; 10: 22.

24. Zamiri M, Jury CS, Dawe RS, et al. Reactivity to autologous serum skin test and relationship with complement levels in chronic idiopathic urticaria and angio-oedema. Clin Exp Dermatol 2009; 34: 587-90.

25. Kikuchi Y, Kaplan AP. A role for C5a in augmenting IgG-dependent histamine release from basophils in chronic urticaria. J Allergy Clin Immunol 2002; 109: 114-8.

26. Ritchie RF, Palomaki GE, Neveux LM, et al. Reference distributions for complement proteins $\mathrm{C} 3$ and C4: a practical, simple and clinically relevant approach in a large cohort. J Clin Lab Anal 2004; 18: 1-8. 